\title{
Innovation braucht Resourceful Humans Aufbruch in eine neue Arbeitskultur durch Virtual Engineering
}

\author{
Jivka Ovtcharova, Polina Häfner, Victor Häfner, Jurica Katicic und \\ Christina Vinke
}

\section{Einleitung und Begriffsklärung}

Der Trend zu mehr sozialem Wohlbefinden im Einklang mit der Wohlstandssteigerung und die verstärkte Rolle der sozialen Vernetzung birgt deutlich die Anzeichen einer tiefgreifenden gesellschaftlichen Veränderung. Dabei handelt es sich nicht mehr nur darum, die Weltwirtschaft für die großen Herausforderungen zu stärken. Menschen mit der Fähigkeit des vernetzten Denkens und Handelns und mit dem Blick für das große Ganze sind gefragt. Bisher wird der Mensch mit seinem Potential, trotz aller Beteuerungen, jedoch noch als „Human Ressource“ aber nicht wirklich als „Resourceful Human“ (in Anlehnung an Fischer 2012) betrachtet. Der Übergang zum „Mensch im Mittelpunkt der Betrachtung“ setzt eine zukunftsfähige Innnovationskultur voraus, die ein grundlegend verändertes Verständnis der menschlichen Möglichkeiten und Bedürfnisse im Umgang mit Technologien, Arbeitssystemen und natürlichen Ressourcen aber auch den Menschen selbst benötigt. Weiterhin sind neue Modellierungs- und Interaktionsparadigmen, Technologielösungen sowie Arbeitskulturen gefragt, die den Wechsel der Blickrichtung zu Innovation durch „Resourceful Humans“ ermöglichen und somit maßgeblich zur effektiven und effizienten Teamarbeit in unternehmensübergreifenden und interkulturellen Unternehmenspartnerschaften beitragen.

Dass Computersysteme dabei eine wichtige Rolle einnehmen, ist unbestreitbar. Im Unterschied zu den traditionellen digitalen Technologien, bei denen die Computersysteme dem Mensch lediglich Hilfestellung anbieten und dieser nach wie vor die Prozesse lenkt, erfordert der interaktive und kreative Mensch-Maschine-Umgang neue Ingenieurmethoden, -inhalte und Kommunikationswerkzeuge, die unter dem Begriff Virtual Engineering

Der Originaltext dieses Beitrags wurde überarbeitet. Das vollständige Korrekturverzeichnis finden Sie am Ende des Buchs und online unter http://dx.doi.org/10.1007/978-3-662-45915-7_16.

J. Ovtcharova $(\varangle) \cdot$ P. Häfner · V. Häfner · J. Katicic · C. Vinke Institut für Informationsmanagement im Ingenieurwesen (IMI), Karlsruher Institut für Technologie (KIT), Karlsruhe, Germany

e-mail: jivka.ovtcharova@kit.edu

A. Botthof, E.A. Hartmann (Hrsg.), Zukunft der Arbeit in Industrie 4.0,

DOI 10.1007/978-3-662-45915-7_12 


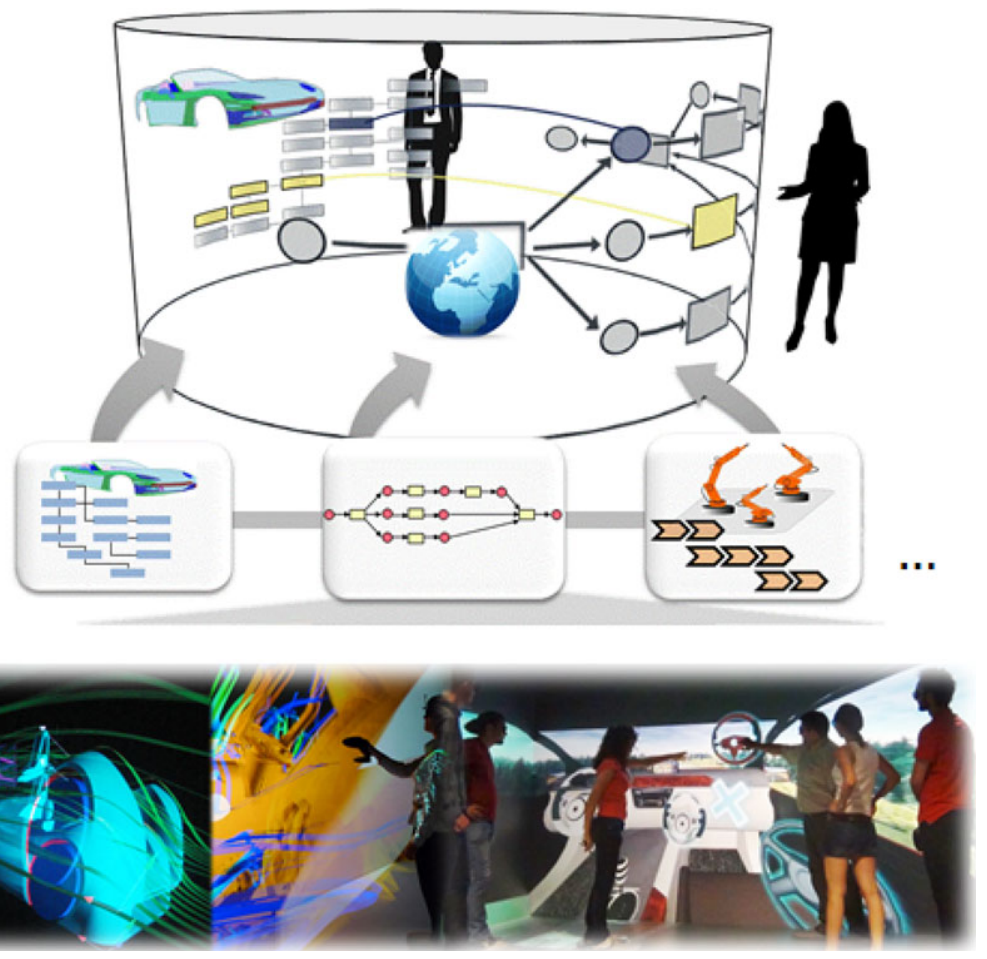

Abb. 1 Begriff des Virtual Engineering, Quelle: Eigene Darstellung

(VE) zusammengefasst werden. So bietet das Virtual Engineering eine integrierte ProzessSystem-Sicht auf das Ganze und ermöglicht unter anderem Entwicklern, Lieferanten, Herstellern und Kunden gleichermaßen, physisch noch nicht existierende Gegenstände rein virtuell zu handhaben und hinsichtlich deren Eigenschaften und Funktionen realitätsnah und ganzheitlich zu beurteilen (Abb. 1).

Was unter dem Begriff „Virtual Engineering“ zu verstehen ist, lässt sich leicht durch Paraphrasieren eines bekannten Zitats von Edsger W. Dijkstra (niederländischer Informatiker) erklären. Er hat einst gesagt: „Informatik hat etwa so viel mit Computern zu tun, wie Astronomie mit Teleskopen“. Dieser Satz bedeutet unter anderem, dass der Computer ein Werkzeug für die Arbeit des Informatikers ist, was oft aus den Augen verloren wird. Das Virtual Engineering hat in derselben Weise etwa so viel mit der virtuellen Realität zu tun, wie Informatik mit Computern. Trotzdem wird das Virtual Engineering in den allermeisten Fällen sofort mit dem Werkzeug ,virtuelle Realität“ assoziiert und nicht mit dem Gedankengut und den Konzepten, die die Ingenieurwissenschaften ausmachen. Dieses Bild reduziert dann leider immer wieder die Bedeutung und das Potenzial des Virtual Engineering einseitig auf äußerliche, rein visuellen Aspekte. Die Zielsetzung des Virtual Engineering ist die Verschmelzung von physischen und virtuellen (Computer-generierten, begehbaren) Wirklichkeiten, Wahrnehmung und Kommunikation durch Täuschung der menschlichen 


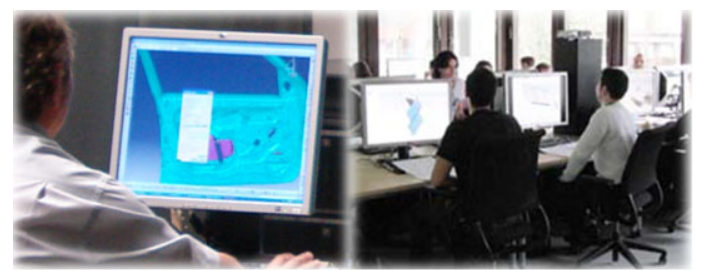

Digital ENGINEERING

- Einzelarbeitsplatz

- Aufgabenorientiert

- IT im Mittelpunkt

- Offline-Anwendung

- GUI-basierte Interaktion

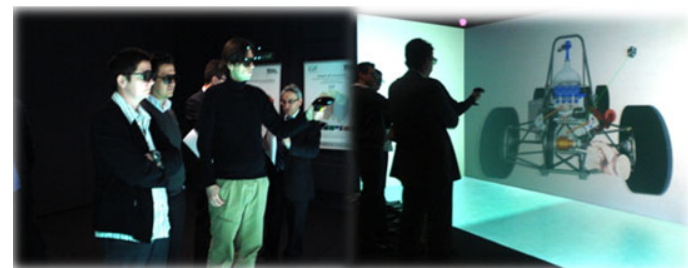

\section{VIRTUAL ENGINEERING}

- Teamarbeitsplatz

- Entscheidungsorientiert

- Mensch im Mittelpunkt

- Echtzeit-Anwendung

- Intuitive Interaktion

Abb. 2 Virtuelles Engineering vs. Digitales Engineering, Quelle: Eigene Darstellung

Sinne in Echtzeit und im Raum und der Einsatz neuer Ingenieurmethoden für realitätsnahe Human-Computer-Interaktion (logisch, nachvollziehbar, intuitiv) in Echtzeit. Damit unterscheidet sich der Ansatz des Virtual Engineering vom traditionellen Ansatz des Digital Engineering wesentlich, wie auf Abb. 2 dargestellt.

Durch den VE-Ansatz sind gegenwärtige Entwicklungs- und Produktionsabläufe grundlegend zu überdenken. So werden Nano- und Mikrostrukturen sowie mechatronische Komponenten eingesetzt, um die Synergien verschiedener Disziplinen wie Mechanik, Elektrik, Elektronik und Software produktiv auszuschöpfen. Eine immer größere Bedeutung erlangen dabei die Cyber-Physischen Systeme (CPS). Nach der Bezeichnung dieses Begriffs bestehen Cyber-Physische Systeme aus verschiedenen vernetzten mechanischen, elektronischen und Softwarekomponenten, die sich selbständig über eine gemeinsame Dateninfrastruktur (z. B. das Internet) untereinander koordinieren (in Anlehnung an Geisberger und Broy 2012). Weiterhin sind moderne 3D- und 4D-Visualisierungstechnologien in Vormarsch. Diese helfen unsichtbare Phänomene sichtbar und frühzeitig validierbar zu machen um dadurch neue Produkteigenschaften und -funktionen zu verwirklichen. Durch den VE-Einsatz werden zukünftige, physisch noch nicht existierende Gegenstände realitätsnah und bedienungsgerecht erlebbar.

Dieser Artikel befasst sich mit der Problematik der Mensch-Maschine-MenschSchnittstelle im Kontext des Virtual Engineering. Da systemtechnische Lösungen heutzutage in Zusammenhang mit der Verwaltung von komplexen und umfangreichen Problemstellungen und Informationsmengen stehen, setzt das Virtual Engineering mit seinem Ansatz „Reducing Complexity“ an und reduziert die Komplexität auf das Wesentliche, um Menschen als „Resourceful Humans“ in Entscheidungsprozessen zu unterstützen. Dies ermöglicht es Menschen, einander an ihren Ideen teilhaben zu lassen und neue Arbeitsumgebungen zu schaffen, in denen multidisziplinäre Teams mit unterschiedlichen, jedoch sich ergänzenden Erfahrungen nachhaltig zusammen arbeiten können. Diese Thematik betrifft über Prozesse der operativen Ebene hinausgehend insbe- 
sondere auch Unternehmensentwicklungs-, Strategieplanungs- und Managementprozesse. Aktuelle VE-Anwendungen in der Produktentwicklung, Produktion und Bildung werden anhand von Forschungsergebnissen des Instituts für Informationsmanagement im Ingenieurwesen (IMI) (www.imi.kit.edu) am Karlsruher Institut für Technologie (KIT) (www. kit.edu) illustriert.

\section{Aufbruch in eine neue Lebens- und Arbeitskultur}

Betrachtet man die Wandlungsprozesse der nächsten fünf bis zehn Jahre (im Sinne der Trendforschung) sind diese durch eine totale Durchdringung der Informations- und Kommunikationstechnologien (IKT) in allen Lebens- und Arbeitsbereichen unserer Gesellschaft gekennzeichnet. So vielseitig und leistungsfähig die IKT-Lösungen auch sein können stehen von nun an die Menschen im Mittelpunkt der Betrachtung. An der ersten Stelle dieses Wandels ist die wachsende Rolle des Individuums zu nennen, mit seiner subjektiven und emotional betonten Wahrnehmung von Produkten, Dienstleistungen oder Lebens-, Bildungs- oder Berufsarten allgemein. Diese resultiert aus der sinkenden Abhängigkeit des Individuums von traditionellen Bindungen und Normen, verstärkt durch den allgemeinen Wohlstandszuwachs seit den 1960er Jahren. Das heißt, die Formen des Zusammenlebens sind immer weniger Ergebnis gesellschaftlicher Zwänge und Vorgaben, sondern Resultat eigenständiger Wahlentscheidungen der Menschen und ihrer Wünsche. So entwickelt sich eine neue Vielfalt von Lebensformen und -stilen, aber auch eine neue Form sozialer Gemeinschaft. Dies geht mit entsprechend veränderter Besitz- und Benutzmotivation einher; Emotion, Erlebnis- und Begeisterungsaspekte treten in den Vordergrund. Immer mehr Menschen gehen engagiert vor, teilen Bilder und Inhalte, kommentieren Aktionen in sozialen Netzwerken, sprechen Weiterempfehlungen aus und fühlen sich bestimmten Marken, Produkten und Dienstleistungen gegenüber verbunden, d. h. sie betrachten diese gewissermaßen als „Freunde“. Der Mensch als Individuum tritt dabei in einer oder mehreren „Rollen“ gleichzeitig auf, u. a. als Produzent, Dienstleister, Kunde oder Wissensempfänger.

Der wahrhaftige Wandel der Gesellschaft von IKT- zu menschzentriert drückt sich zuallererst in einem natürlichen Mensch-Maschine-Kommunikationsstil aus, der auf Verständnis und Dialog zwischen Mensch und Maschine basiert und die Verknüpfung von mehreren Kommunikationskanälen der menschlichen Wahrnehmung (unter anderem Sehen, Hören, Riechen, Schmecken und Tasten) voraussetzt. Noch steht dieser Wandel und der damit verbundene Aufbruch in eine neue Lebens- und Arbeitskultur ganz am Anfang, jedoch schon heute sind weitgehende Auswirkungen in allen menschlichen Lebensbereichen, wie Gesellschaftsformen, Technologie, Ökonomie und Wertesysteme sichtbar.

\section{Qualitativ neue Ansätze für die gute Arbeit in der Industrie 4.0}

Im Institut für Informationsmanagement im Ingenieurwesen am Karlsruher Institut für Technologie wurde 2008 ein Lifecycle Engineering Solutions Center (LESC) (www.lesc. kit.edu) mit dem Ziel eröffnet, neueste Ergebnisse aus der Forschung und Virtual Engi- 
neering Best Practices in industrielle Anwendungen nahtlos zu transferieren. In diesem Zentrum stehen zu Zwecken der Anwendungs-, Grundlagenforschung und der Lehrtätigkeiten des Instituts skalierbare high-end VE-Systeme zur Verfügung. Zahlreiche Aktivitäten werden durchgeführt, beginnend mit der Entwicklung einer Virtual-Reality-Engine „PolyVR“ über den Aufbau von Virtual Mock-Ups im Produktlebenszyklus und die interaktive Flexibilitätsbewertung in der Produktion über die Erforschung des emotionalen Kundenfeedback für variantenreiche Produkte und die Schaffung neuer Formen des Wissenserwerbs in immersiven Umgebungen. Im Folgenden werden ausgewählte Ansätze des Instituts vorgestellt.

\section{Skalierbare Virtual Engineering Anwendungen}

Betrachtet man den heutigen Stand der Arbeit und Technik ist es festzustellen, dass trotz des Einsatzes moderner Technologien, u. a. der virtuellen Realität, das „Windows, Icons, Menus, Pointer (WIMP)“-Paradigma der 80er Jahre unseren Alltag immer noch bestimmt. Abgeleitet aus diesem Kenntnisstand ergibt sich die zentrale Fragestellung des Virtual Engineering: Inwieweit trägt die Weiterentwicklung der VR-Technologien zu einer Veränderung der Mensch-Maschine-Interaktion als Voraussetzung einer natürlichen und intuitiven Form der Kooperation von Menschen mit Maschinen bei? Hierbei soll in einem ersten Schritt untersucht werden, inwieweit der Mensch seine Intuition und natürlichen Instinkte zur Interaktion mit der Maschine verwenden kann. Dabei geht es um sein Gesamtverhalten, wie z. B. seine Körperhaltung, Gestik und Sprache. So setzt der Mensch seinen ganzen Körper zur Interaktion in einer VR-Umgebung ein. Er nimmt Informationen mit seinen fünf Sinnen gleichzeitig auf und reagiert mit Sprache, Handlungen und unbewusster Körpersprache auf diese. Das Ziel des Virtual Engineering ist es daher, dieses breite Spektrum menschlicher „Ein-und Ausgangskanäle“ mit Hilfe von VR-Systemen zu simulieren, um sich der vollkommenen Interaktion anzunähern. Dann lernt der Mensch nicht mehr wie er Systeme bedient, sondern das System lernt wie es dem Menschen dient, was heutzutage eine der größten Herausforderungen des Wechsels zu „Resourceful Humans“ darstellt.

Die virtuelle Realität wird dabei als ein Medium bezeichnet, „... das aus interaktiven Rechensimulationen besteht, welche die Lage und Handlungen des Teilnehmers verfolgen und das Feedback zu einem oder mehreren menschlichen Sinnen ersetzen oder erweitern, so dass der Teilnehmer das Gefühl hat, mental immersiv oder präsent in der Simulation zu sein“ (Sherman und Craig 2003). Bei den angesprochenen menschlichen Sinnen handelt es sich meist um das Sehen (i.d.R. 3D-Projektion), das Hören (i.d.R. räumlicher Schall) und den Tastsinn (haptische Geräte). Technologien wie Head- oder Finger-Tracking ermitteln in Echtzeit die Lage und Orientierung von Teilen des Nutzerkörpers und passen die Simulation an seine Aktion an. Der Nutzer kann mit Hilfe von Eingabegeräten oder Gesten durch die virtuelle Welt navigieren und sie manipulieren. Die drei Haupteigenschaften der virtuellen Realität sind (Burdea und Coiffet 2003): 
- Immersion als Grad der Eingebundenheit des Nutzers in der virtuellen Welt

- Interaktion als bidirektionaler Informationsfluss zwischen dem Nutzer und der virtuellen Welt

- Imagination als Ausmaß des Vorstellungsvermögens des Nutzers, angeregt durch die Darstellung

Eine mittels VR-System erzeugte virtuelle Welt, die diese Haupteigenschaften aufweist, wird als immersive Umgebung bezeichnet. Industrielle Anwendungen der virtuellen Realität, mit Ausnahme einiger wirtschaftsstarker Branchen wie Automobil- und Flugzeugindustrie, konnten sich jedoch kaum in der Breite durchsetzen. Dies lag vor allem an den hohen Anschaffungs- und Wartungskosten der Hardware- und Software und oft nicht ausgereiften Insellösungen. Ein wichtiger Paradigmenwechsel, der in den letzten Jahren in der Spieleindustrie zu beobachten war, findet dagegen auch im Bereich des Ingenieurwesens Beachtung. Neue Interaktionswerkzeuge, wie beispielsweise Microsofts Kinect ${ }^{\mathrm{TM}}$ oder LeapMotion ${ }^{\mathrm{TM}}$ zur Gestensteuerung, sind als ausgereifte Produkte mit klaren Konzepten auf den Markt gekommen.

Wichtige Ansichten sind in diesem Zusammenhang die Interaktion, Intuition und Imagination. Die Desktop-Umgebung wird als klassische Mensch-Maschine-Schnittstelle durch neuartige Systeme abgelöst, in denen der Nutzer die zentrale Rolle spielt. Statt ein System zu bedienen, soll der Mensch intuitiv die visualisierten Inhalte erfahren und verändern können. Um in die virtuellen Welten eintauchen zu können, müssen die Schnittstellen hoch immersiv gestaltet sein. Diese Bedingung kann von den aktuell verfügbaren Technologien in der Unterhaltungsindustrie - 3D-Fernsehern, kostengünstigen HeadMounted-Displays und Spielekonsolen - erfüllt werden, die natürliche Interaktionen wie Gestenerkennung nutzen. Die Vorteile dieser Konzepte ergeben sich durch die wesentlich schnellere Übertragung von Informationen an den Nutzer, da sich dieser mit seinen Sinnen viel präsenter und eingebundener fühlt. Daraus folgen eine kürzere Einarbeitungszeit, eine schnellere Interaktion und ein besseres Einschätzungsvermögen.

Die im Institut entwickelte Virtual Reality Software PolyVR adressiert die o. g. Herausforderungen, indem sie skalierbare und schnell generierbare VE-Systemlösungen unterstützt. Sie ist die ideale Lösung zum Erstellen und Erleben von interaktiven 3DApplikationen. So wird der Import unterschiedlichster Daten erleichtert, wie zum Beispiel 3D-Inhalte in Form von Numerik-, Netz-, Volumen- oder CAD-Daten. Zusätzlich können auch Animationen, Ton und physikalische Echtzeitsimulationen in die VR-Szene eingebunden werden (Abb. 3).

Wichtigste Eigenschaften von PolyVR sind dabei die flexible Konfiguration und einfache Einbindung aller gängigen VR-Ein- und Ausgabegeräte wie Trackingsysteme und Computer-Cluster für verteilte Visualisierung. Headtracking ermöglicht die ständige Anpassung der Nutzerperspektive und hat einen noch wesentlich größeren Einfluss auf die Tiefenwahrnehmung als die stereoskopischen Darstellungen. Die 3D-Anwendungen sind von den Hardware-Systemen entkoppelt, dies erlaubt einen flexiblen Einsatz der Applikation auf allen unterstützenden Hardware-Systemen. Die dadurch erreichte Skalierbarkeit 

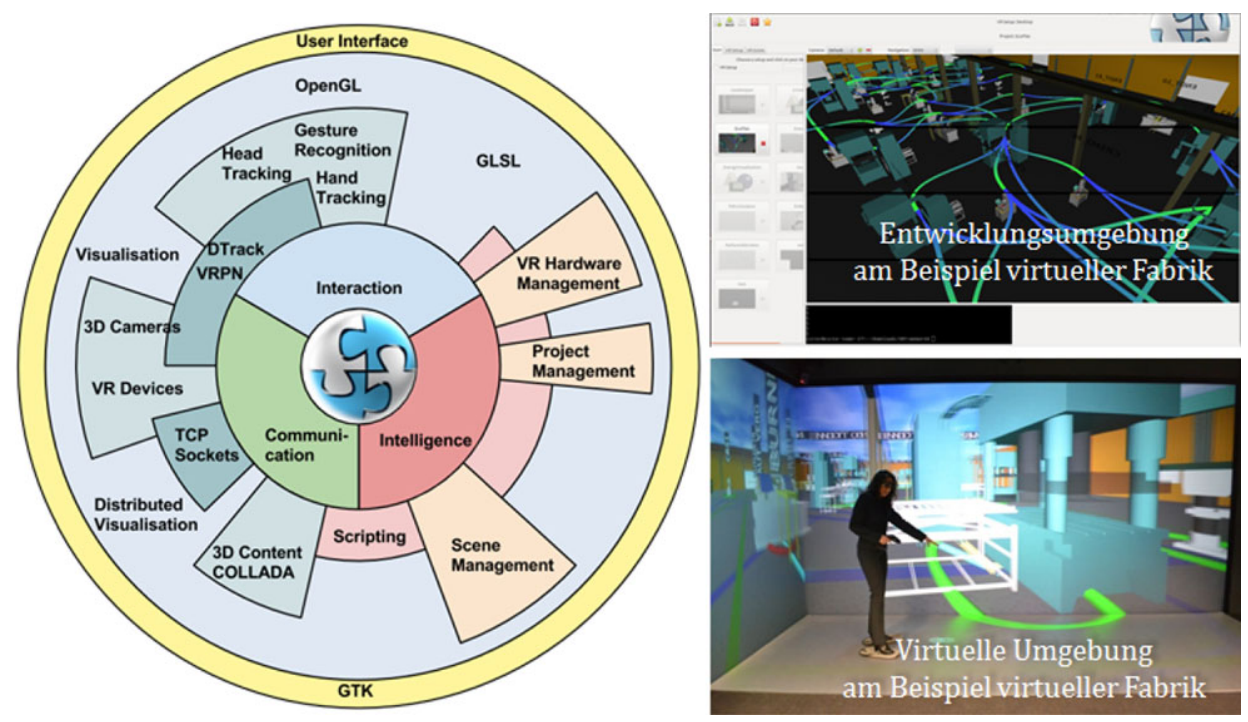

Abb. 3 Software-Architektur und Arbeitsumgebungen der PolyVR-Software, Quelle: Eigene Darstellung

von Lösungen erlaubt somit den Einsatz nicht nur in hoch immersiven VR-Umgebungen wie CAVEs oder Holospaces, sondern ebenso auf KMU-gerechten VR-Umgebungen, z. B. unter Einsatz von 3D-Fernsehern.

Weiterhin bietet die intuitive Benutzeroberfläche eine einfache Bedienung, auch für nicht VR-Experten. Interaktive 3D-Inhalte können an der laufenden Anwendung verwaltet, verändert und erweitert werden. Eine intuitive Darstellung des Szenengraphen erlaubt jeden Aspekt der Szene wie 3D-Modelle, Licht, Kameras oder Interaktion schnell zu verändern. Die in PolyVR integrierten umfangreichen Möglichkeiten Skripte zu erstellen, sind für Entwickler ein bequemes Werkzeug zur Implementierung komplexer Funktionalität.

\section{Flexibilitätsmanagement in der Produktion}

Die Vorteile der VE-Technologie in der Produktentwicklung und Produktion wurden relativ früh wie folgt erkannt (Ovtcharova et al. 2005):

- Integration von Simulationsmodellen aus unterschiedlichen Ingenieursdisziplinen

- Modellierung und Validierung von für den Nutzer wahrnehmbaren Produkteigenschaften, die schwer explizit spezifizierbar sind

- Effiziente Erfassung von Nutzerpräferenzen und Anforderungen

- Vorausschauende Simulation und Beurteilung von Produktkonzepten, die erst in der Zukunft durch technologische Fortschritte realisierbar werden 
Abb. 4 Eintauchen in die virtuelle Fabrik, Quelle: Eigene Darstellung

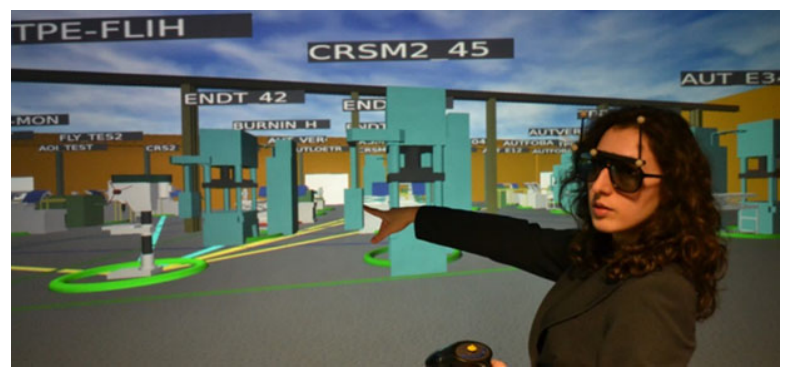

Zur Bewältigung der Herausforderung speziell in der Produktion wurde am Institut der Methodenbaukasten ecoFLEX (Rogalski und Wicaksono 2012) entwickelt. Auf Basis eines Bewertungskonzepts für Produktionsressourcen werden Energie-, Personal-, Materialund Betriebsmittelbedarfe sowohl aus strategischer Sicht als auch im operativen Einsatz geplant. Über eine mitgelieferte Funktionalität zur Flexibilitätsanalyse lassen sich zudem bestehende Schwächen bei der Auslegung, Dimensionierung und Personalbelegung von Produktionsanlagen bewerten. Die Kopplung von ecoFLEX mit der bereits beschriebenen PolyVR-Engine erlaubt ein immersives Eintauchen in die virtuelle Arbeitsumgebung von Produktionsanlagen (Abb. 4).

Dadurch erhalten Produktionsplaner nunmehr die Möglichkeit, die mit ecoFLEX erkannten Ineffizienzen im Ressourceneinsatz detailliert an den virtuellen Anlagemodellen zu untersuchen und Lösungsalternativen zu erarbeiten, was sonst nur am realen Objekt erreichbar wäre. Mit Hilfe der virtuellen Abbildung der Produktionsmittel und der Simulation von Prozessen ist es möglich, unterschiedliche Bearbeitungsstrategien auf ihre Auswirkungen auf das Gesamtsystem hin zu testen. Zu diesem Zweck stehen vielfältige Interaktionsmöglichkeiten und Analysefunktionen bereit. Diese erleichtern es maßgeblich, komplexe Zusammenhänge und verknüpfte Informationen aufzunehmen und im Sinne eines verbesserten Anlagenbetriebs bereits in den Phasen der Neu- und Änderungsplanung anzuwenden. Somit werden unnötige Mehrkosten bedingt durch einen ineffizienten Ressourceneinsatz im Live-Betrieb oder weniger sinnvolle Anpassungen der Produktionsinfrastruktur vermieden und finanzielle Spielräume für zukünftige Investitionen im Anlagenbau geschaffen. Der oben beschriebene Lösungsansatz integriert die interaktive VR-Umgebung als Frontend mit eigenständigen Simulationstools im Backend. Diese wird mit einer bidirektionalen Netzkommunikation realisiert, die nachhaltige und erweiterbare Schnittstellen gewährleistet.

\section{Erfassung und Bewertung von emotionalem Kundenfeedback}

Der Markterfolg eines Unternehmens hängt maßgeblich von der Zufriedenheit seiner Kunden ab (Homburg und Bucerius 2006). Aus diesem Grund ist eine kontinuierliche Einbindung der Endkunden in den Produktentwicklungsprozess sehr wichtig. Das Prinzip des Frontloading besagt aber, dass gerade in den frühen Phasen ein verstärkter Einsatz von 


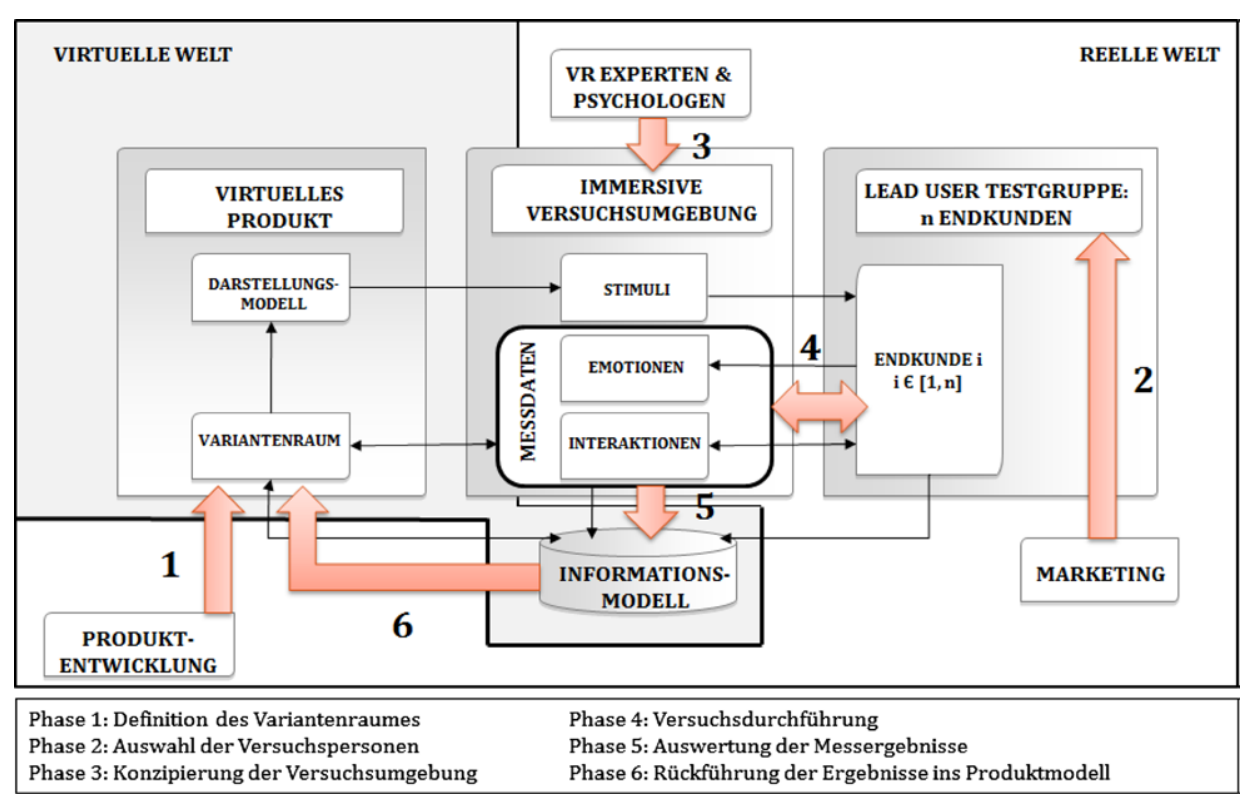

Abb. 5 Sechs Schritte der Methodik EMO VR, Quelle: Eigene Darstellung

Ressourcen zur Erwerbung von Wissen über das zukünftige Produkt sinnvoll ist, denn zu diesem Zeitpunkt verfügt das Unternehmen über einen großen Entscheidungsspielraum und kann auch Änderungen zu relativ niedrigen Kosten durchführen (Ovtcharova 2010). Also bringt die Kundeneinbindung gerade in den frühen Phasen des konzeptuellen Designs von virtuellen Produkten den größten Vorteil. Da Emotion als eine der drei Erfolgsparameter des modernen Marketings identifiziert wurde (Kreutzer und Merkle 2006) und Endkunden größtenteils und zunehmend emotionale Kaufentscheidungen treffen, muss insbesondere das emotionale Kundenfeedback über virtuelle Produkte erfasst werden. Aktuell werden Kunden in der Regel nicht in der Ideenauswahlphase eingebunden, sondern erst zum späteren Zeitpunkt. Somit erreicht die große Mehrheit der Designideen gar nicht den Endkunden.

Am IMI wurde eine neuartige Methodik (Katicic 2012) entwickelt, um frühzeitig zuverlässiges emotionales Kundenfeedback über Produkte zu erhalten, die sich in den frühen Phasen der Entwicklung befinden und daher nur virtuell existieren. Das Akronym dieser Methodik lautet EMO VR und geht auf die Technologien der Emotionserkennung und der Virtuellen Realität zurück, deren einzigartige Kombination dieser Methodik zugrunde liegt. Sie besteht aus sechs Schritten (Abb. 5). Im Laufe der Durchführung sind Iterationen sinnvoll und notwendig. Der Erfolg der Methodik hängt entscheidend von der ebenbürtigen Beteiligung unterschiedlicher Experten (Ingenieure, Designer, Marketingexperten, Psychologen, Virtual-Reality-Fachleute) ab. 
Abb. 6 EMO VR

Versuchsumgebung, Quelle:

Eigene Darstellung

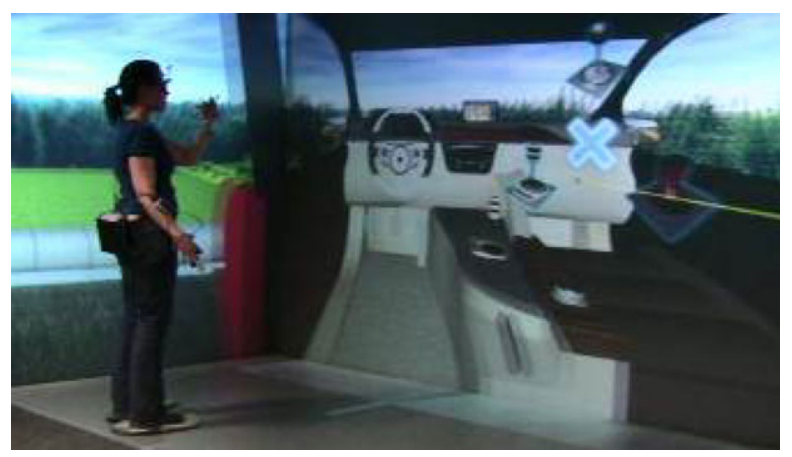

Zur Validierung der vorgestellten Methodik wurde eine Studie mit 21 Teilnehmern am Lifecycle Engineering Solutions Center (LESC) durchgeführt, indem die Versuchsumgebung anhand der Feststellungen (Ovtcharova und Katicic 2011) ausgewählt wurde. Die PolyVR-Engine wurde bei der Implementierung des Anwendungsfalls - ein Konfigurator für ein Fahrzeugcockpit - eingesetzt. Zur Visualisierung wurde eine passive Drei-WandStereoprojektion mit den Abmessungen 4,5 $\mathrm{m} \times 2,6 \mathrm{~m} \times 1,9 \mathrm{~m}$ benutzt. Das optoelektronische Trackingsystem der Firma ART wurde für das Head-Tracking und zur Interaktion mit der Anwendung eingesetzt (Abb. 6). Zur Interaktion mit der virtuellen Welt wurde eine einfache Geste „Pont\&Click“ implementiert (Bewegung vor- und rückwärts schnell nacheinander in Richtung des gezielten Objektes). Die peripheren physiologischen Messungen erfolgten mit dem mobilen Gerät NEXUS-32. Aufgenommen wurden Signale der Gesichtsmuskeln (EMG), die elektrodermale Aktivität der Finger (EDA) und der Puls (BVP) auf dem Mittelfinger der inaktiven Hand.

Die zwei modernen Technologien der Virtuellen Realität und der Emotionserkennung wurden in die parallel verlaufenden verketteten Prozesse der Produktentwicklung und Marktforschung so integriert, dass aus einer Vielfalt alternativer zukünftiger Produktdesigns die emotional ansprechenden (funktionsfähigen) Designs aus der Sicht des Endkunden, und somit im Sinne der Gesellschaft, identifiziert werden. Immersive Umgebungen ermöglichen eine kontexttreue Darstellung des virtuellen Produktes im realen Maßstab, wodurch das Vorstellungsvermögen des Kunden und die natürliche Abgabe seines emotionalen Feedbacks begünstigt werden. In diesen Umgebungen ist zuverlässiges emotionales Feedback nur dann erfassbar, wenn bereits eine individuelle emotionale Kalibrierung zur Referenzbildung durchgeführt wurde und wenn die implementierte Interaktion routinemäßig erfolgt und somit die Emotionen des Kunden nicht beeinträchtigt. Die Erfassung peripherer physiologischer Signale wurde als vielversprechend bestätigt, während geschlossene Fragen eine unzureichende Wiederholbarkeit der Ergebnisse aufgrund der verfälschenden kognitiven Vermittlung lieferten. Die vorgeschlagene Methodik ist kohärent und erzielt Synergien zwischen Experten aus unterschiedlichen Fachgebieten. 


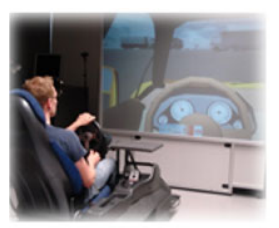

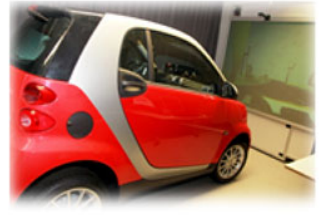

Mixed Reality Driving Simulator

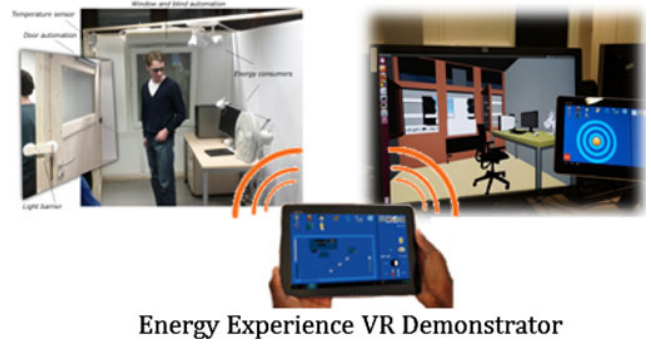

Abb. 7 Beispiele für immersive Lernumgebungen, Quelle: Eigene Darstellung

\section{Wissenserwerb in immersiven Lernumgebungen}

Immersive Umgebungen werden zunehmend für Bildungs- und Trainingszwecke eingesetzt. Diese sogenannten virtuellen Lernumgebungen haben das Ziel, den Nutzern Wissen und praktische Fähigkeiten zu vermitteln (Abb. 7). Die anschauliche und anwendungsbezogene Darstellung von Lerninhalten in virtuellen Lernumgebungen trägt gerade bei komplexen oder abstrakten Themen zu einem größeren Verständnis des Lernstoffs bei. Des Weiteren bieten virtuelle Lernumgebungen die Möglichkeit des direkten Erlebens von Lerninhalten und können mit Hilfe von Individualisierung und Selbststeuerung des Lernens zu einer Steigerung des Lernerfolgs führen. Ein weiterer Vorteil von virtuellen Lernumgebungen ist die Simulation wirklichkeitsgetreuer Bedingungen bei Trainings, welche in der Realität mit hohen Gefahren oder Kosten verbunden sind.

Am IMI wurde der Einfluss unterschiedlicher Immersionsgrade und verschiedener Bewegungsarten zur Navigation in virtuellen Umgebungen auf die menschliche Gedächtnisleistung erforscht (Häfner et al. 2013). Mit Hilfe eines simplen Gedächtnistests ist die menschliche Gedächtnisleistung in einer hoch-immersiven CAVE und einer niedrig immersiven virtuellen Desktop-Umgebung in Bezug auf unterschiedliche Bewegungsarten quantifiziert worden. Als Bewegungsarten sind physisches und virtuelles Gehen untersucht worden. In der CAVE wurde den Probanden unter Zuhilfenahme einer Motion-CapturingTechnik die Navigation mittels aktivem physischem Gehen ermöglicht. Die Verwendung des Navigationsgerätes ART Flystick2 ${ }^{\mathrm{TM}}$ befähigte die Probanden zum aktiven virtuellen Gehen in der CAVE. Aus technischen Gründen war in der virtuellen Desktopumgebung keine Navigation durch Motion-Capturing möglich. Den Ergebnissen zufolge konnte ein Einfluss der Bewegungsart auf die menschliche Gedächtnisleistung weder bewiesen noch widerlegt werden.

Des Weiteren lieferte die ANOVA hinsichtlich des Einflusses des Immersionsgrades von virtuellen Umgebungen auf die menschliche Gedächtnisleistung einen p-Wert von 0,101. Da dieser Wert knapp oberhalb der Grenze eines marginal signifikanten Ergebnisses liegt, wird angenommen, dass ein Effekt in der Grundgesamtheit besteht. Das erfordert aber eine Eruierung durch Folgestudien. Des Weiteren sollen in Zukunft lernbezogene Aufgaben mit höheren kognitiven Belastungen untersucht werden. Aus den Ergebnissen der Studie wurden folgende Interaktionsparadigmen für den VR-Bereich formuliert: 

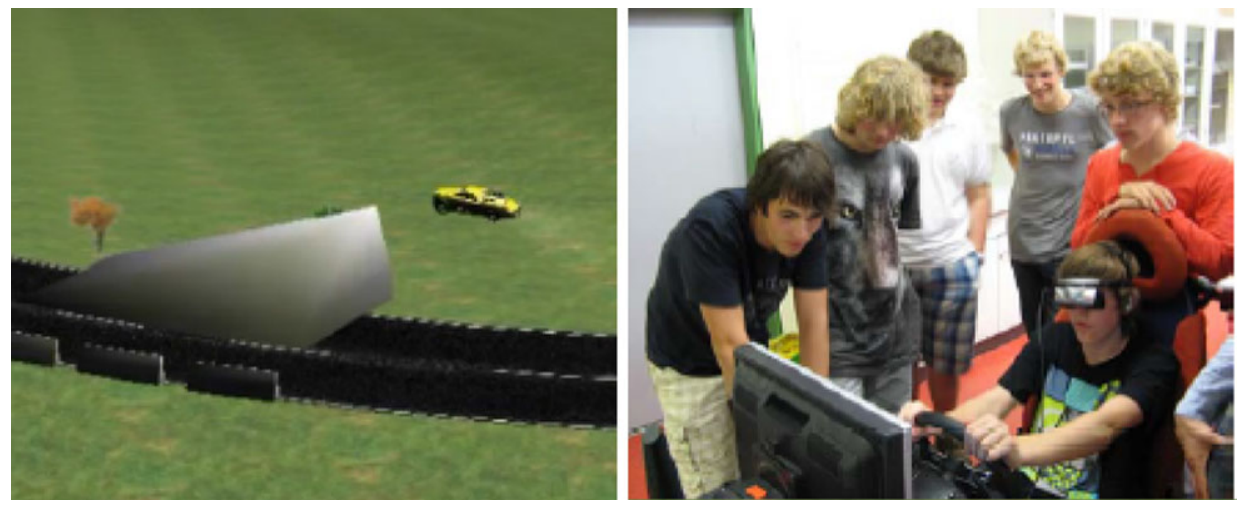

Abb. 8 Virtual Reality Fahrsimulator als immersive Lernumgebung, Quelle: Eigene Darstellung

- Die Bewegungsart in einer virtuellen Umgebung beeinflusst wahrscheinlich nicht die menschliche Gedächtnisleistung.

- Der Grad der Immersion einer virtuellen Umgebung beeinflusst sehr wahrscheinlich die menschliche Gedächtnisleistung.

Ein weiteres Beispiel zum Einsatz von immersiven Lernumgebungen ist das im Rahmen des von der Baden-Württemberg-Stiftung finanzierten Projektes „MINT-Box DRIVE“ (Häfner et al. 2014). Die Schüler des Einstein-Gymnasiums und der Tulla-Realschule in Kehl haben sich mit physikalischen Gesetzen der Themengebiete Regelkreise, Energie und Mechanik spielerisch auseinandergesetzt. Mit visuellen Programmieren und Parameteränderungen konnten sie die Auswirkung von physikalischen Größen untersuchen und, unterstützt durch moderne Virtual Reality Lösungen, am Fahrsimulator live erleben (Abb. 8).

Der Experimentierkasten besteht aus einem Fahrersitz, dem Logitech G27 System (Lenkrad, Pedalen und Schaltung) und Head Mounted Displays als Ausgabe und stellt eine intuitive Mensch-Maschine-Schnittstelle dar. Die Schüler konnten auf diese Weise durch Vernetzung von Denken, Handeln und Intuition Fähigkeiten zum Erkennen und Lösen von interdisziplinären Problemen aus dem Gebiet der MINT-Fächer entwickeln. Im Unterricht wurden vorrangig Methoden zum individuellen und selbstbestimmten Lernen eingesetzt. Durch das Nutzen von Virtual-Reality-Systemen wurde bewiesen, dass das naturwissenschaftlich-technische Interesse bei SchülerInnen durch kreative Arbeit und spielerisches Lernen gefördert werden kann. Die modernen VR-Lösungen unterstützen das räumliche Vorstellungsvermögen und erzeugen eine nachhaltige Weiterbildungsmotivation im naturwissenschaftlich-technischen Bereich.

Die Entwicklung von virtuellen Umgebungen im Bildungsbereich erfordert ein tiefgreifendes Verständnis der technologischen und inhaltlichen Aspekte, die den Lernprozess und das Verständnis des Nutzers fördern und unterstützen. Dabei sind die Vorteile von virtuellen und besonders von immersiven Lernumgebungen gegenüber traditionellen Lehrmetho- 
den hinsichtlich ihrer inhaltlichen und technologischen Relevanz nur rudimentär erforscht (Schwan und Buder 2006).

\section{Ausblick}

Die vorgestellten Forschungsergebnisse zeigen auf, dass die Virtual Engineering-Methoden in der Zukunft eine immer bedeutendere Rolle bei der Produktentwicklung und Produktion sowie beim Lernen spielen werden. Der Erfolg im Übergang von technologie- zu menschgerechten Arbeitssystemen, Produkten und Dienstleistungen in Industrie 4.0 hängt sehr stark vom Innovationsgrad eines Unternehmens ab. Ökonomisch entscheidend ist ein hoher Innovationsgrad bei geringen Kosten und kompromissloser Qualität. Aufgrund der Wertschöpfung durch Einsatz intelligenter Komponenten und Cyber-Physischen Systemen sind innovative und Mensch-zentrierte Produkte jedoch von hoher interdisziplinärer und damit komplexer Natur. Hierzu ist eine starke Kopplung eines physischen und eines Computermodells nötig. Diese Kopplung stellt die ultimative informationstechnische Basis des Virtual Engineering dar, die das daraus resultierende Zusammenwachsen realer und virtueller Welten ingenieurmäßig in weitreichende und alle Lebensbereiche durchdringende Lösungen umsetzt.

Open Access This chapter is distributed under the terms of the Creative Commons Attribution Noncommercial License, which permits any noncommercial use, distribution, and reproduction in any medium, provided the original author(s) and source are credited.

\section{Literaturverzeichnis}

Burdea, G. C., \& Coiffet, P. (2003). Virtual reality technology (S. 1-3). New York: Wiley Interscience.

Fischer, H. (2012). Der Sohn will die Revolution. Human Resources Manager, 2012, 45-47.

Geisberger, E. \& Broy, M. (Hrsg.) (2012). AgendaCPS, integrierte forschungsagenda, cyberphysical systems, acatech studie.

Häfner, P., Vinke, C., Häfner, V., Ovtcharova, J., \& Schotte, W. (2013). The impact of motion in virtual environments on memorization performance. In 2013 IEEE international conference on computational intelligence and virtual environments for measurement systems and applications (CIVEMSA) (S. 104-109). New York: IEEE.

Häfner, P., Häfner, V., \& Ovtcharova, J. (2014). Experiencing physical and technical phenomena in schools using virtual reality driving simulator. In P. Zaphiris (Hrsg.), LNCS: Bd. 8524. Humancomputer interaction, Part II, HCII 2014 (S. 50-61). Switzerland: Springer.

Homburg, C., \& Bucerius, M. (2006). Kundenzufriedenheit als Managementherausforderung. In Kundenzufriedenheit: Konzepte - Methoden - Erfahrungen (S. 64-65). Wiesbaden: Gabler.

Katicic, J. (2012). Methodik für Erfassung und Bewertung von emotionalem Kundenfeedback für variantenreiche virtuelle Produkte in immersiver Umgebung. Dissertation am Karlsruher Institut für Technologie (KIT).

Kreutzer, R. T., \& Merkle, W. (2006). Die Notwendigkeit zur Neuausrichtung des Marketing. In Die neue Macht des Marketing (S. 13-17). Wiesbaden: Gabler. 
Ovtcharova, J., Weigt, M., \& Seidel, M. (2005). Virtual Engineering - Handlungsbedarf und Lösungsansätze zur Prozess- und Systemintegration Virtuelle Produkt- und Prozessentwicklung. In: Magdeburger Maschinenbau-Tage. Tagungsband 7.

Ovtcharova, J. (2010). Vorlesungen im Fach Virtual Engineering. Wintersemester: Karlsruher Institut für Technologie. 2010/2011.

Ovtcharova, J., \& Katicic, J. (2011). Design of immersive environment for capturing of emotions to virtual products. In Wissenschaftliche Konferenz „Innovationen und Wettbewerbsfähigkeit“, Sofia (S. 203-208).

Rogalski, S., \& Wicaksono, H. (2012). Methodology for flexibility measurement in semi-automatic production. In H. A. ElMaraghy (Hrsg.), Enabling manufacturing competitiveness and economic sustainability (S. 141-146). Berlin: Springer.

Sherman, W. R., \& Craig, A. B. (2003). Understanding virtual reality: interface, application, and design (S. 5-6). San Francisco: Morgan Kaufmann.

Schwan, S., \& Buder, J. (2006). Virtuelle Realität und E-Leaning. 